heitsgurt" oder adjuvante antiarrhythmische Therapie implantiert werde.

\section{Auswurffraktion kann sich erholen}

Studien zufolge kann sich nicht nur nach einem frischen Infarkt, sondern auch nach einer Bypass-OP und bei nicht ischämischen Kardiomyopathien (vor allem bei Myokarditis) die Auswurffraktion in den folgenden Monaten bei fast jedem Zweiten zumindest teils erholen. Eine ICD-Implantation ist dann nicht mehr gerechtfertigt. „Es empfiehlt sich daher bei solchen Patienten immer eine Kontrolle der EF nach vier bis sechs Monaten und eine Überbrückung mit einer Defibrillator-Weste, ehe die endgültige Entscheidung für die ICD-Implantation getroffen wird“, so Klein. Auch bei einer kardialen Synkope sollte eine reversible
Ursache immer ausgeschlossen sein. Bei Patienten mit terminaler Herzinsuffizienz kann der akute Herztod durchaus ein gnädiges Ereignis darstellen. Hier stellt sich die Frage, ob und wann der ICD abgestellt werden darf. „Diese normative Entscheidung muss immer im Einzelfall mit Einbeziehung des Patienten getroffen werden“, sagte Klein.

Dr. Peter Stiefelhagen

\title{
Synkopenabklärung - Anamnese als Schlüssel zur Diagnose
}

\begin{abstract}
Die Abklärung einer plötzlichen Bewusstlosigkeit ist eine häufige wie auch schwierige Herausforderung. Vorrangig geht es immer darum, eine kardiale Ursache nachzuweisen oder auszuschließen. Um unnötige Schrittmacherimplantationen zu vermeiden, ist eine fokussierte Diagnostik zu empfehlen.
\end{abstract}

U nter einer Synkope versteht man einen vorübergehenden Bewusstseinsverlust infolge einer globalen zerebralen Ischämie; das „Umfallen“ bedeutet also noch lange nicht "Synkope“. "Zunächst geht es bei jedem Patient, der ,umgefallen' ist, darum, andere Ursachen der Bewusstlosigkeit etwa einen Krampfanfall auszuschließen", sagte Prof. Dietrich Andresen von den Elisabeth-Kliniken in Berlin. Die häufigsten Ursachen einer echten Synkope seien die Orthostase, eine reflektorische Störung und eine kardiale Rhythmusstörung.

\section{Keine Schrotflintendiagnostik}

Das Spektrum der apparativen Untersuchungen zur Abklärung der Synkope ist breit. Es umfasst das EKG, Belastungs-

EKG, Langzeit-EKG, den Schellong-Test, die Kipptisch-Untersuchung, Loop- bzw. Event-Recorder ebenso wie EEG, MRT und CCT. „Doch der diagnostische Wert

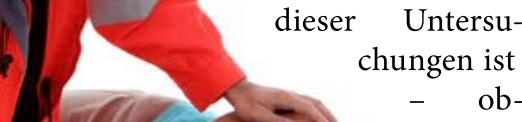

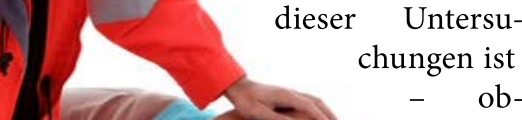

Es gibt viele Gründe für eine Bewusstlosigkeit. Für ein rhythmogenes Ereignis spricht das „sofortige Umfallen“. wohl sie viel, ja zu viel eingesetzt werden - sehr gering“, berichtete Andresen. Sinnvoller sei es, fokussiert und nicht mit einem

diagnostischen Rundumschlag vorzugehen. Bei jedem zweiten Patienten liefere allein die ausführliche Anamnese schon die richtige Diagnose. Prämonitorische Symptome wie Unwohlsein oder Schwäche in den Beinen sprechen für eine reflektorische Synkope. Ein sofortiges Umfallen hingegen weist eher auf eine Rhythmusstörung hin. Auch eine Synkope im Sitzen sei immer dringend verdächtig auf ein rhythmogenes Ereignis.

Wegen der prognostischen Bedeutung ist der Nachweis bzw. Ausschluss einer kardialen Ursache vorrangig. So gehört zur unverzichtbaren Basisdiagnostik neben dem EKG auch eine Echokardiografie. Im Allgemeinen wird ein LangzeitEKG abgeleitet. Doch nicht selten zeigt sich hier ein Befund, der nichts beweist und nichts ausschließt wie eine Asystolie mit einer Dauer unter drei Sekunden oder eine nur sehr kurz anhaltende Tachykardie. „Wenn dieser Befund nicht mit Symptomen einhergeht, ist damit keinesfalls die rhythmogene Ursache be-

wiesen, sondern man darf dann eine solche nur annehmen “, erklärte Andresen.

Nur wenn EKG-Befund und Symptome korrelieren, sei eine entsprechende Therapie mit Schrittmacher oder ICD gerechtfertigt. Wenn aber während der Symptomatik keine Rhythmusstörung dokumentiert werden könne, sei eine kardiale Synkope ausgeschlossen. Bei rezidivierenden Synkopen und Verdacht auf eine kardiale Ursache sollte man heute ein Ereignis-Recorder implantieren. „Ein solcher Recorder ist kosteneffektiv, da er unnötige Krankenhausaufenthalte verhindern kann“, so Andresen.

\section{Zu viele Schrittmacher implantiert?}

Der Karotisdruck ist nicht empfehlenswert, um einen hypersensitiven Karotissinus nachzuweisen. „Diese Untersuchung ist so unspezifisch, dass ich sie in meiner Klinik verboten habe“, so Andresen. Eine Schrittmacherindikation bestehe nur bei einem Karotissinus-Syndrom, also wenn entsprechende Symptome bei Drehbewegungen des Kopfs oder Einengungen des Halses auftreten.

Das unsachgemäße Vorgehen bei Patienten mit einer Synkope ist nach Meinung von Andresen einer der Gründe, warum in Deutschland zu viele Schrittmacher implantiert werden. „Wir implantieren jährlich 80.000 Schrittmacher, ich glaube, 50.000 würden reichen.“

Auch eine im EKG dokumentierte Asystolie müsse nicht immer zwangsläufig zu einer Schrittmacherimplantation führen. Gehe der Asystolie nämlich eine langsame Frequenzabnahme voraus, sei dies der typische Befund einer vagovasalen Synkope und solche Patienten benötigten keinen Schrittmacher.

Quelle: Hannover Herz Messe, 8.5.2015 